\title{
UNE NOUVELLE ESPÈCE DU GENRE TEUCRIUM SECTION POLIUM, ENDÉMIQUE DU MAROC: TEUCRIUM MITECUM IBN TATTOU \& EL OUALIDI
}

\author{
Jalal EL OUALIDI et Mohammed IBN TATTOU
}

\begin{abstract}
RÉSUMÉ.Une nouvelle espèce du genre Teucrium Section Polium, endémique du Maroc: Teucrium mitecum Ibn Tattou \& El Oualidi. Les auteurs décrivent une nouvelle espèce et donnent sa répartition et son écologie. Les caractères de ce nouveau taxon sont discutés par rapport à ceux de certaines espèces affines notamment $T$. buxifolium Schreb., T. musimonum Humbert et T. huotii J. Emb. \& Maire.
\end{abstract}

Mots clés. Teucrium section Polium, T. mitecum, endémique , Maroc.

ABSTRACT. A new species of the genus Teucrium L. Section Polium endemic of Morocco: Teucrium mitecum Ibn Tattou \& El Oualidi. A new specie of the genus Teucrium L. section Polium (Lamiaceae) is described. The authors give also its ditribution and ecology. The characters of the new taxa are discussed comparatively with those of affined species, particulary T. buxifolium Schreb., T. musimonum Humbert and T. huotii J. Emb. \& Maire.

Key words. Teucrium section Polium, T. mitecum, endemic, Morocco.

\section{INTRODUCTION}

Dans une prospection de la terminaison nord-orientale du Moyen Atlas (Mitek, fig. 1), l'un de nous a récolté des échantillons qui paraissaient à première vue se rapprocher de Teucrium buxifolium Schreb. et de Teucrium thymifolim Schreb. L'examen des échantillons de l'Herbier RAB a révélé que la plante présentait également des affinités avec d'autres taxons : T. musimonum Humbert (endémique du Maroc) et $T$. rotundifolium Schreb. (endémique d'Espagne et du Maroc). Cependant le T. mitecum s'en distingue bien par des caractères propres.
Teucrium buxifolium, était connu jusqu'à présent par T. buxifolium ssp buxifolium, ssp. rivasii (Rigual ex Greuter \& Burdet) Crespo et T. buxifolium ssp.hifacense (Pau) Fernández Casas de l'Espagne méridionale, (Willkomm \& Lange, 1870 ; Tuttin ; Heywood, 1963 et Crespo et al., 1990) et par la T. buxifolium ssp. albidum (Munby) Greuter \& Burdet de l'Algérie occidentale, en Oranie, (Munby, 1855) jusqu'à la frontière marocaine (Ghar Roubane, fig. 1). De ce fait, il a toujours été recherché au Maroc et l'on considérait l'Atlas Saharien comme une aire probable pour ce taxon (Cohen, I956). 


\section{RESULTATS}

Teucrium mitecum M. Ibn Tattou \& J. El Oualidi, sp. nov.

A Teucrio buxifolio Schreb. habitu, foliorum paginis discoloribus calycisque forma accedit. Ab eo autem discrepat foliorum caulinorum supernorum limbo orbiculari, ovali vel reniformi basi troncato raro cuneato (nec anguste ovali subdeltoideo oblongo basi cuneato) ; calycis dentibus non perspicue ciliatis, pilos adpressos antrorsos illis tubi non longiores margine ferentibus (nec pilis erectis illis tubi longioribus conspicue ciliatis); floralibus bracteis flores plerumque superantibus, 1-2 dentibus utraque margine crenatis (necflores non superantibus, supernis integris); pilis complanatisfaucis tubi corollae plus minusve latioribus.

Holotypus : Maroc, Moyen Atlas (MA), plaine de Mitek entre jbel Ouarirt et jbel Lahmar à environ $900 \mathrm{~m}$. steppe d'Alfa et de Romarin, le 9-IV-1991, Ibn Tattou M. (RAB 61811). Exemplaire du bas. Isotypus : RAB (61814). Des échantillons isotypus ont été deposés aux herbiers: MGC (36233), MPU et GDAC.

Illustration : figure 2.
- Description morphologique:

Plante vivace, petite, atteignant $7 \mathrm{~cm}$ de long, à souche ligneuse peu épaisse, émettant de nombreuses tiges grêles, \pm ligneuses à leur base, étalées sur le sol ou \pm procombantes (fig. 2). Tige couverte de poils tecteurs blancs, courts, courbés, \pm apprimés-antrorses et peu denses (fig. 3a). Feuilles inférieures très petites, à limbe d'environ $2 \times 2 \mathrm{~mm}$, puis augmentant de taille jusqu'à l'inflorescence; les supérieures à limbe atteignent $7(-10) \times 6(-8) \mathrm{mm}$, parfois plus larges que longues (fig. 4a); feuilles à limbe orbiculaire, ovale ou réniforme, à sommet arrondi, à base le plus souvent tronquée ou rarement subcordée à \pm cunéiforme; marges \pm révolutées et pourvues de 4-6 crénelures de chaque côté chez les feuilles supérieures ; feuilles inférieures entières ou à marges pourvues de 1-2 crénelures de chaque côté. Pétiole des feuilles inférieures long de 1-1.5 $\mathrm{mm}$, celui des feuilles supérieures long de 2-5 $\mathrm{mm}$; face supérieure des feuilles verte, couverte de poils blancs courbés, apprimés antrorses peu denses ; face inférieure densément tomenteuse et blanchâtre (fig. 2). Bractées florales inférieures identiques aux feuilles

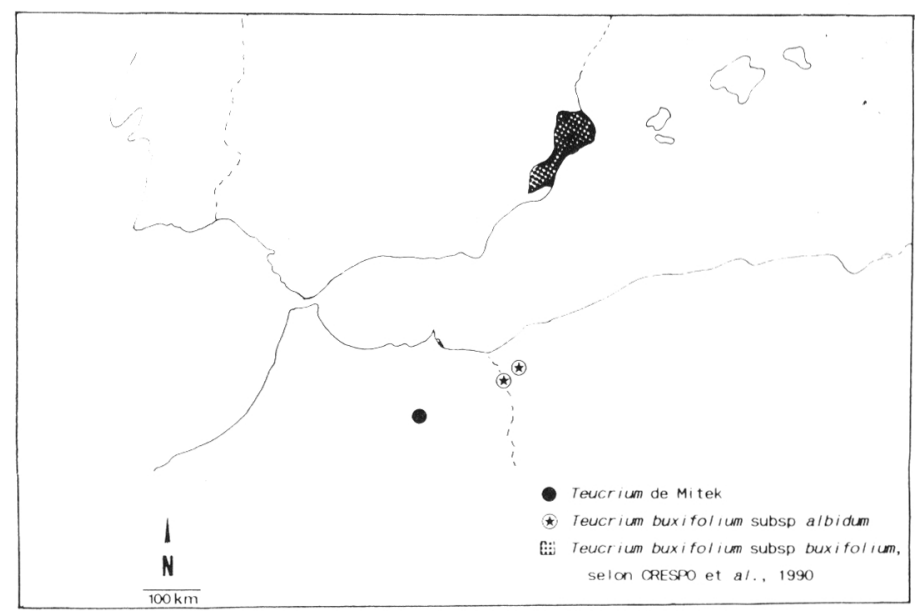

Figure 1. Distribution et localités des Teucrium buxifolium ssp. buxifolium, Teucrium buxifolium ssp. albibum et Teucrium mitecum. 


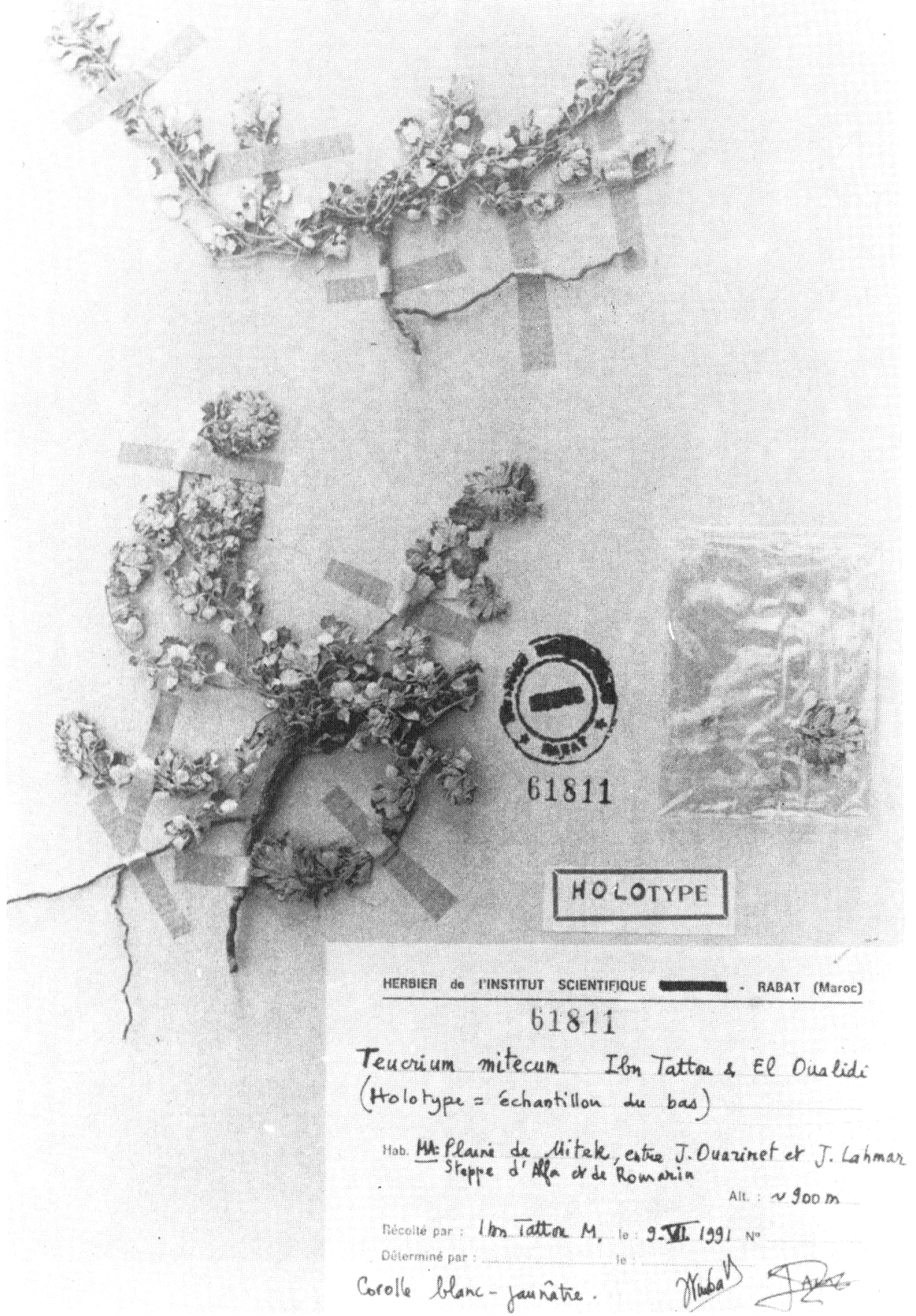

Figure 2. Planche d'herbier RAB illustrant l'holotype de l'espèce Teucrium mitecum. 


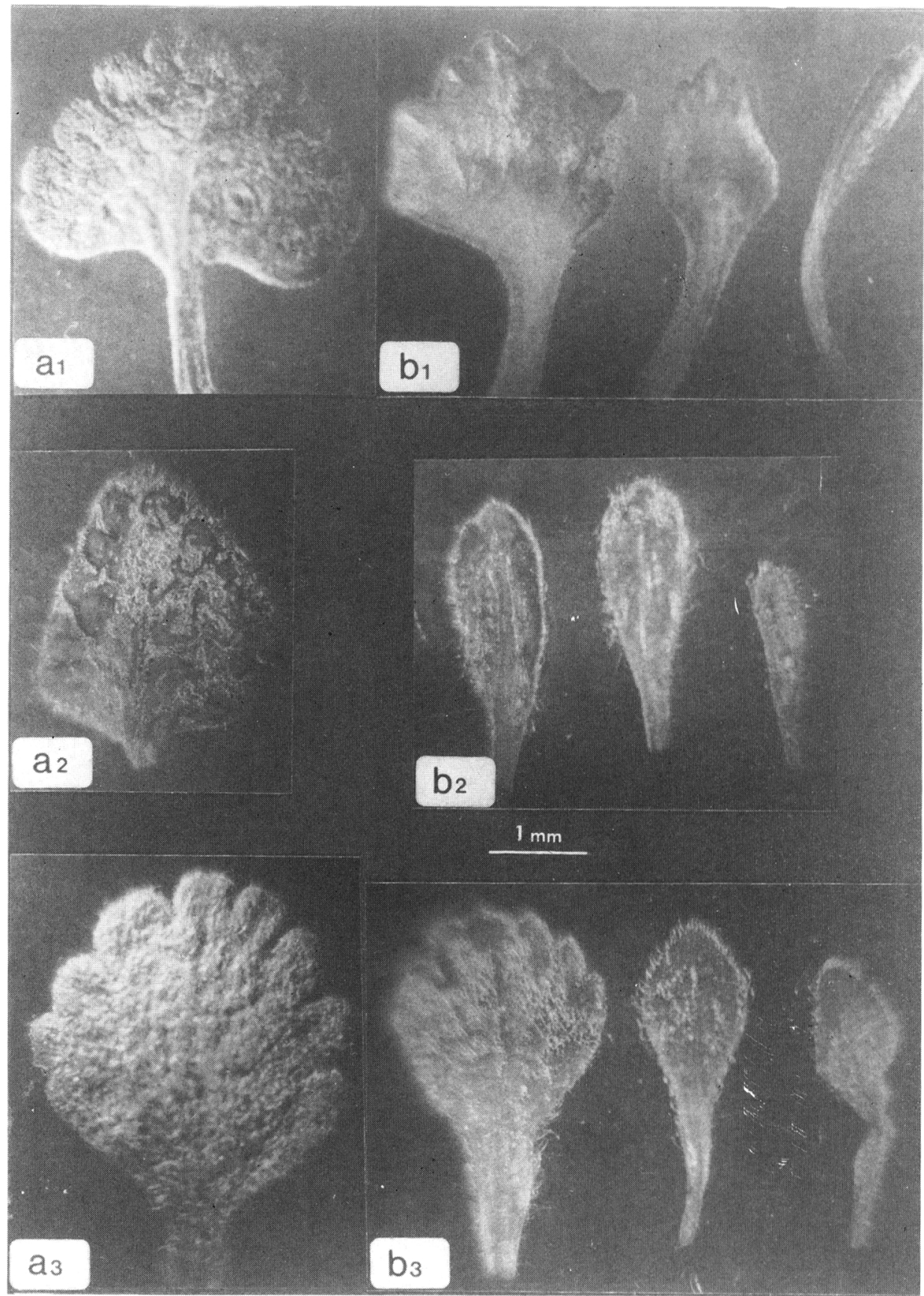

Figure 3. Détails en microscopie photonique de l'indument de la tige et de la pilosité de la gorge de la corolle: Tige (a1: Teucrium mitecum, a2: T. buxifolium ssp. buxifolium, a3: T. buxifolium ssp. albidum; corolle (b1: T. mitecum, b2: T. buxifolium ssp. buxifolium b3: T. buxifolium ssp. albidum). 
caulinaires supérieures, les autres de plus en plus réduites, les extrêmes ovales à ovaleslancéolées, à base atténuée-cunéiforme, habituellement plus longues que les calices ou les égalant; à marges portant 1-2 crénelures de chaque côté (fig. 4b). Inflorescence en grappes contractées au sommet. Fleurs, 2 par noeud, groupées en grappes d'abord condensées en têtes puis devenant allongées et \pm lâches ; pédicelles floraux longs d'environ $1 \mathrm{~mm}$; calice campanulé, $6-8 \times 4 \mathrm{~mm}$; à base étroite et \pm arquée ; à dents longues de $1.25-1.5 \mathrm{~mm}$, à la fin divergentes; à tube parcouru par 10 nervures, anastomosées sur la moitié supérieur du tube et au niveau des dents; nervure centrale de chaque dent plus ou moins saillante sur une grande longueur en carène et se terminant au sommet en pointe \pm acuminée ; calice intérieurement glabrescent à légèrement poilu, couvert sur la face externe de poils blancs, courts, mous, courbés-antrorses et peu denses ; dents à marges pourvues de poils apprimés courts jamais dressés ni étalés-dressés de sorte que les dents paraissent non ciliées (fig. 5); corolle longue de 10-11 mm, jaunâtre ou blancjaunâtre, à tube long d'environ $5 \mathrm{~mm}$ et plus court que le calice ou l'égalant; lobe antérieur environ $4 \times 3.5 \mathrm{~mm}$, à sommet \pm anguleuxarrondi ; lobes latéraux de $2.5 \times 1 \mathrm{~mm}$; lobes latéro-postérieurs d'environ $4 \times 1.5 \mathrm{~mm}$, à marges courtement ciliées. Face externe couverte de poils tecteurs épars et de poils glanduleux sessiles et pluricellulaires ; face interne \pm poilue et pourvue au niveau de la gorge de 2 rangées de poils aplatis lancéolés, de 1.2-1.6x0.35-0.6 mm, contrastant par leur grosse taille avec le reste de la pilosité (fig. 3b) ; androcée, typique des Teucrium, à 4 étamines, les inférieures plus longues et à anthères plus grandes que les supérieures; ovaire et style glabres; akène à texture alvéolée du type $T$. polium.

Ecologie et répartition:

La plante a été récoltée d'abord en bas de pente du jbel Lahmar en exposition ouest, vers 900 m d'altitude, sur sol marno-argileux, sous un lambeau de pinède (Pinus halepensis Miller). Elle a été ensuite trouvée toujours à la même altitude dans la plaine de Mitek, entre jbel Lahmar et jbel Ouarirt, dans une steppe de Romarin et d'Alfa, sur le même type de sol dont l'ambiance bioclimatique se situerait dans l'aride supérieure ou le semi-aride inférieur.

Cette région qui est constituée par les reliefs nord-est les plus extrêmes du Moyen Atlas, jouxtant du côté oriental la vaste plaine de Guercif à bioclimat plus aride, est un carrefour biogéographique qui abrite une quantité importante de plantes endémiques, marocaines (telle que Pitardia nepetoides Batt.), algéro-marocaines (Tripodion kremerianum (Cosson) Lassen, Genista spartioides subsp. pseudoretamoides Maire), et hispano-algéro-marocaines (Trigonella ovalis Boiss., Silene oropediorum Cosson, etc.).

\section{DISCUSSION ET CONCLUSION}

Sur la base de la valeur diagnostique testée de certains caractères (El Oualidi \& Puech, 1993), Teucrium mitecum appartient incontestablement à la section polium. A l'intérieur de cette section elle se rapproche notamment aux Teucrium: T. buxifolium, $T$. musimonum et $T$. huotii.

La comparaison de T. mitecum avec celles d'Espagne (RAB 29504, MGC 34259 et 6951), et d'Algérie (RAB s.n.) montre (tab. 1) qu'elles ont en commun :

La pilosité au niveau des feuilles, avec la face supérieure verte et peu poilue, la face inférieure densément poilue blanchâtre, des poils aplatis au niveau de la gorge du tube de la corolle, la nervation au niveau du calice avec les nervures centrales des dents plus ou moins saillantes, en carène longitudinale.

Mais si la $T$. mitecum se rapproche au $T$. buxifolium ssp. buxifolium par la taille et la 


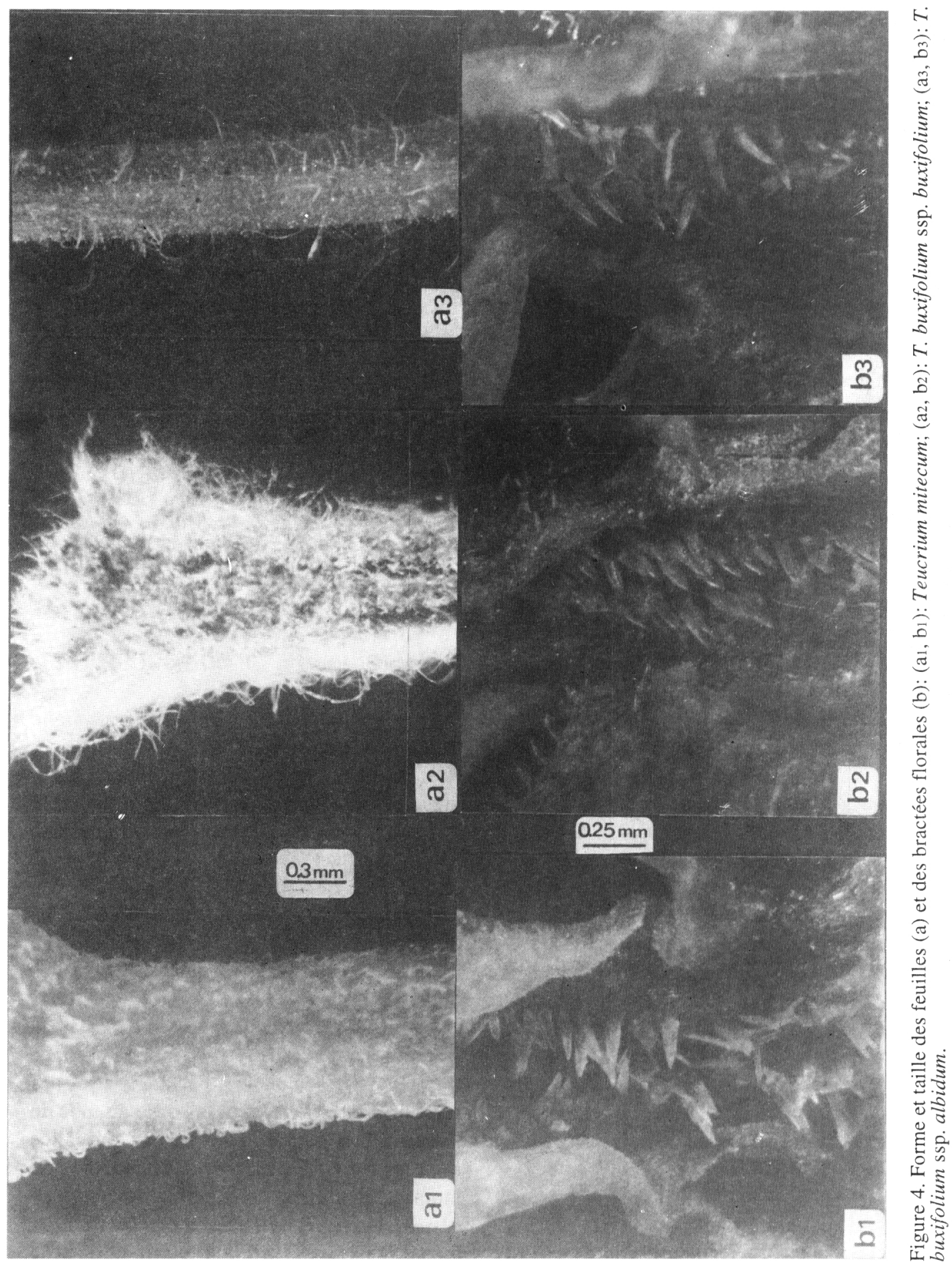




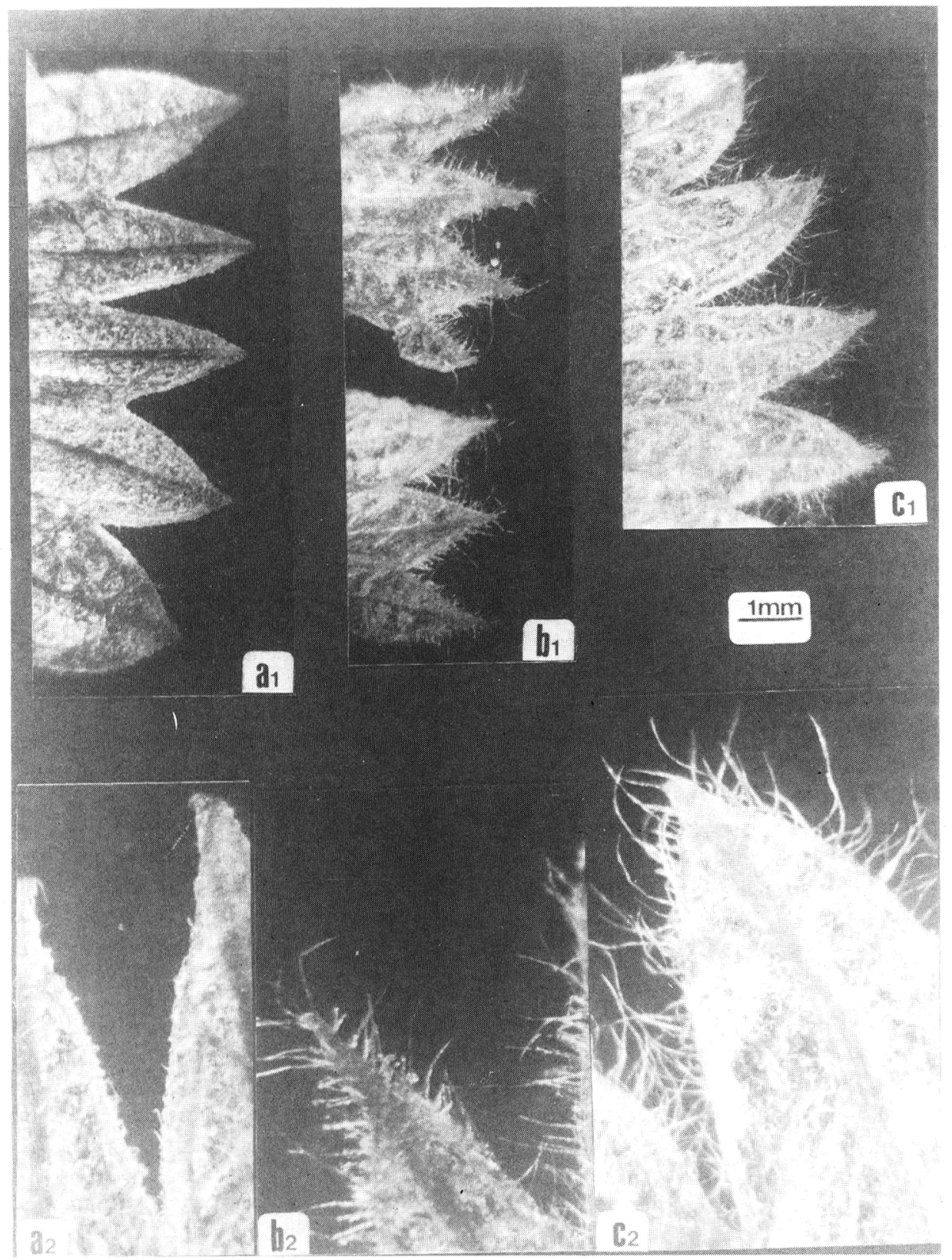

Figure 5. Détails en microscopie photonique de la pilosité au niveau des dents du calice: (a1, a2): Teucrium mitecum; (b1, b2): T. buxifolium ssp. buxifolium; (c1, c2): T. buxifolium ssp. albidum. 
forme du calice, elle s'en éloigne nettement par la forme des feuilles (fig. 4a), la pilosité de la tige (fig. 3a) et du tube du calice et par la couleur de la corolle. La plante marocaine a des feuilles orbiculaires à subréniformes, tronquées à subcordées à la base, des calices et tiges portant des poils mous flexueux et \pm apprimés antrorses peu denses et une corolle blanc-jaunâtre, alors que celle d'Espagne forme des feuilles ovales à oblongues, des tiges couvertes dans la partie supérieure d'un tomento dense blanchâtre, des calices à poils dressés et des corolles roses. En revanche, $T$. mitecum se rapproche au $T$. buxifolium ssp albidum par la même forme des feuilles et la même pilosité au niveau du tube du calice et s'en éloigne par la taille du calice et de la corolle, la pilosité de la tige (fig. 3a) et des dents du calice (fig. 5).

Sur la base des connaissances acquises (Cohen, 1956 ; Sauvage \& Vindt, 1955, 1965) ; Puech, 1976, 1984 ; Navarro, 1988 ; El Oualidi, 1991) sur la valeur diagnostique des caractères concernant la section polium du genre Teucrium, les différences morphologiques mises en évidence sont suffisantes, à notre sens, pour créer Teucrium mitecum. Son existence dans cette région nordest du Moyen Atlas qui concentre un certain nombre de taxons endémiques, plaide pour ce point de vue.

Bien entendu, ce travail descriptif, basé sur des différences morphologiques ne représente qu'une première approche, utile, mais qu'il faudrait appuyer par des recherches biosystématiques, notamment cytologiques et chimiques. Néanmoins nous pensons que les taxons évoqués dans cette étude : T. mitecum, T. buxifolium, T. thymifolium et T. musimonum constituent un même complexe d'espèces récemment différenciées, dans des milieux différents et bien isolés les uns des autres. Leur biogéographie a probablement favorisé un isolement et une spéciation selon le processus classique d'allopatrie. La variation géographique que montre la germandrée de Mitek a été réalisée dans les refuges nord-est du Moyen Atlas, une région intéressante par son écologie et sa biogéographie, et qui représente un véritable foyer d'endémisme.

\section{BIBLIOGRAPHIE}

COHEN, E. -1956- Contribution à l'étude des Teucrium marocains de la section polium. Trav. Inst. Sci. Chérifien. Sér. Bot., 9, 88p.

CRESPO, M.B., G. MATEO \& J. GÜEMES 1990- Sobre Teucrium buxifolium Schreber (Lamiaceae) y especies relacionadas. Bol. Soc. Brot. Ser. 2. 63: 121-131.

EL OUALIDI, J. -I991- Biosystématique et taxinomie des Teucrium de la section Polium (Lamiaceae) dans le bassin méditerranéen occidental. Différents aspects de la variation au Maroc, en France et en Espagne. Thèse Doct., USTL, Montpellier II, $22 \mathrm{O}$.

EL OUALIDI, J. \& S. PUECH -1993- Quelques marqueurs morphologiques des Teucrium section polium du Maroc. Valeurs diagnostiques à différents niveaux d'intégration. Acta Bot. Malacitana, 18:.

TUTIN, T.G. \& D. HEYWOOD -1972- Teucrium buxifolium Schreber, in Flora Europea. vol 3, TUTIN T.G. et al. (Ed.), p 133.

GREUTER, W., H.M. BURDET \& G. LONG (Ed.) -1986- Med-Checklist, 3 Dicot., p 367.

MUNBY, G., 1855- Notice sur quelques plantes d'Algérie. Bull. Soc. Bot. France, vol 2, p. 286.

NAVARRO, T. -1988- Estudios biosistemáticos en el género Teucrium (Sección polium subsección polium) en la Península Ibérica (Lamiaceae). Tesis Doctoral. Universidad de Granada.

NAVARRO, T \& J.L. ROSUA -1990Nomenclatural and taxonomic notes on the Teucrium Section Polium (Miller) Schreber (Lamiaceae) in the Iberian peninsula. Candollea, 45(2): 581-589.

PUECH, S. -1976-Recherches de biosystématique sur les Teucrium (Labiées) de la section polium du bassin méditerranéen occidental (Espagne et France). Thèse Doct. Sci., U.S.T.L., Montpellier, 138p. 


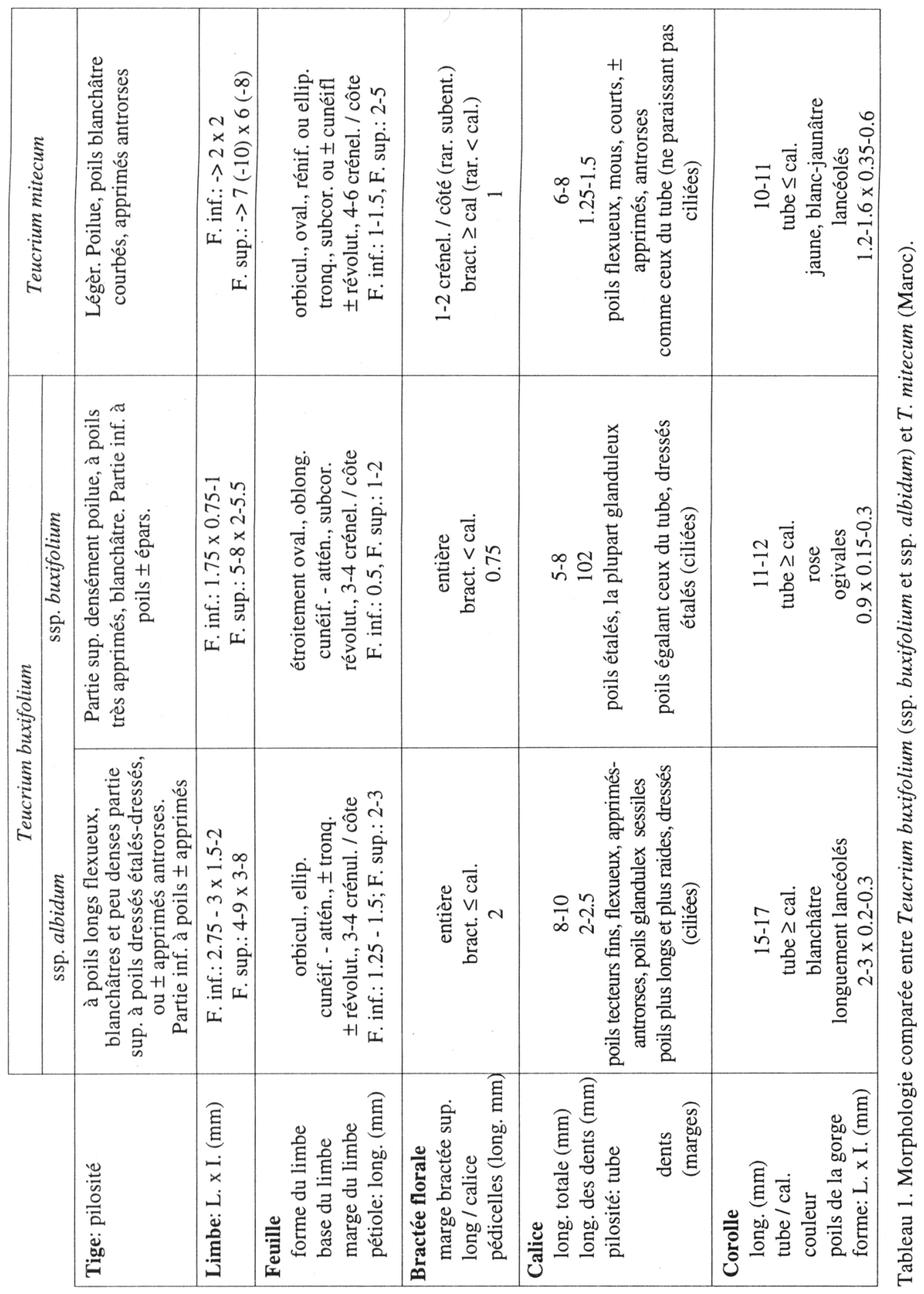


PUECH, S. -1984- Les Teucrium (Labiées) de la sect. polium du bassin méditerranéen occidental (France et Péninsule Ibérique). Naturalia Monsp., A5, 71p.

SAUVAGE, Ch. \& J. VINDT -1955- Synopsis du genre Teucrium Sect. polium au Maroc. Bull. Soc. Sc. Nat. Phy. du Maroc, 35: 283-293.

SAUVAGE, Ch. \& J. VINDT -1965- Synopsis du genre Teucrium Sect. polium au Maroc (suite et fin). Naturalia Monsp., 16: 195-200.

SAUVAGE, Ch. \& J. VINDT -1967-Révision de l'herbier de l'Institut scientifique chérifien. Notes critiques. Le Botaniste, Sér. L: 415-436.
WILLKOMM, M. \& J. LANGE-1870- Prodromus florae hispanicae. Vol 2, Stuttgartiae sumtibus E. Schweizerbart (E. Koch), p: 475-476.

Aceptado para su publicación en Agosto de 1993

Adress des auteurs. Institut Scientifique, Univ. Mohammed V, Rabat, Agdal, BP 703, Maroc. 